\title{
Archéopages
}

Archéopages

Archéologie et société

38 | 07/2013

Terres inhospitalières

\section{Une grande construction néolithique sur poteaux}

\section{Stéphane Blanchet}

\section{OpenEdition}

Journals

Édition électronique

URL : https://journals.openedition.org/archeopages/518

DOI : 10.4000/archeopages.518

ISSN : 2269-9872

\section{Éditeur}

INRAP - Institut national de recherches archéologiques préventives

\section{Édition imprimée}

Date de publication : 1 août 2014

Pagination : 68-69

ISSN : $1622-8545$

\section{Référence électronique}

Stéphane Blanchet, " Une grande construction néolithique sur poteaux 》, Archéopages [En ligne], 38 |

07/2013, mis en ligne le 01 juillet 2015, consulté le 23 janvier 2022. URL : http://

journals.openedition.org/archeopages/518 ; DOI : https://doi.org/10.4000/archeopages.518 


\section{Une grande construction néolithique sur poteaux}

Stéphane Blanchet

Inrap, UMR 6566 "Centre de recherche en archéologie, archéosciences, histoire», responsable d'opération

Site

Les Lignes de la Gonzée

La Mézière

Ille-et-Vilaine

Date

Février-octobre 2012

Surface fouillée

6 ha

Équipe

Étude céramique

David Gâche, Inrap

Étude lithique

Marion Lemée, Inrap
Dominant un petit vallon et implantée sur d'épais placages de lœss remanié, la maison, qui mesure $12 \mathrm{~m}$ de large et s'étend sur au moins $20 \mathrm{~m}$ de long [ill. 1], est construite sur poteaux selon un plan quadrangulaire d'orientation est-ouest.

Malheureusement, elle semble avoir été tronquée par le passage d'une route communale, ce qui complique quelque peu l'analyse architecturale de l'ensemble. À l'est de la route, plusieurs creusements se rattachent à l'occupation néolithique, et il n'est pas exclu que certains d'entre eux appartiennent à l'extrémité orientale de la construction. Elle se développerait alors sur près de 40 m de long [ill. 2]. Cependant, en regard des structures de fondation observées sur la partie occidentale de l'édifice, les différences de comblement et de profil de ces creusements ne permettent pas d'en confirmer totalement l'hypothèse.

L'ossature principale de l'architecture est constituée de poteaux généralement disposés en tierce [ill. 3] Sur une partie de la maison, seuls deux gros poteaux centraux ont été plantés, probablement pour libérer de l'espace interne. En périphérie, de petits trous de poteau matérialisent quant à eux l'emplacement des parois du bâtiment. Les fosses d'implantation des éléments principaux sont assez remarquables puisqu'elles mesurent $1 \mathrm{~m}$ de diamètre pour $1,20 \mathrm{~m}$ de profondeur. Si l'on y ajoute l'épaisseur des niveaux décapés, leur profondeur initiale était de l'ordre de 1,50 m. Les négatifs observés dans les coupes stratigraphiques indiquent l'utilisation de poteaux d'environ $0,50 \mathrm{~m}$ de diamètre.

Une grande partie du mobilier céramique et lithique mis au jour provient d'un lambeau de vieux sol conservé au niveau de la maison ainsi que du comblement des négatifs de poteaux. L'assemblage céramique compte notamment des jattes, des bols, des fragments de coupes à socle. Outre des produits de débitage,

le mobilier lithique comprend quant à lui de l'outillage que l'on retrouve habituellement en contexte domestique, à savoir des grattoirs, des lames de haches, des armatures tranchantes ainsi que des meules et des enclumes. Le mobilier céramique et lithique mis au jour ainsi que les premières datations ${ }^{14} \mathrm{C}$ permettent de rattacher l'ensemble au début du Néolithique moyen II vers 4200 avant notre ère. Les études à venir permettront de préciser comment s'insère le site dans les ensembles culturels de l'ouest de la France et notamment dans le groupe dit de Castellic.

On notera qu'une quinzaine de structures à pierres chauffées [ill. 4] se concentrent à proximité immédiate de la maison. De forme circulaire, elles mesurent environ $1 \mathrm{~m}$ de diamètre et sont construites avec des blocs de quartz associés à quelques fragments d'instruments de mouture.

Le décapage et les fouilles mis en œuvre sur les six hectares n'ont révélé aucun autre édifice de ce type. En revanche, des trous de poteau se rattachant manifestement à des
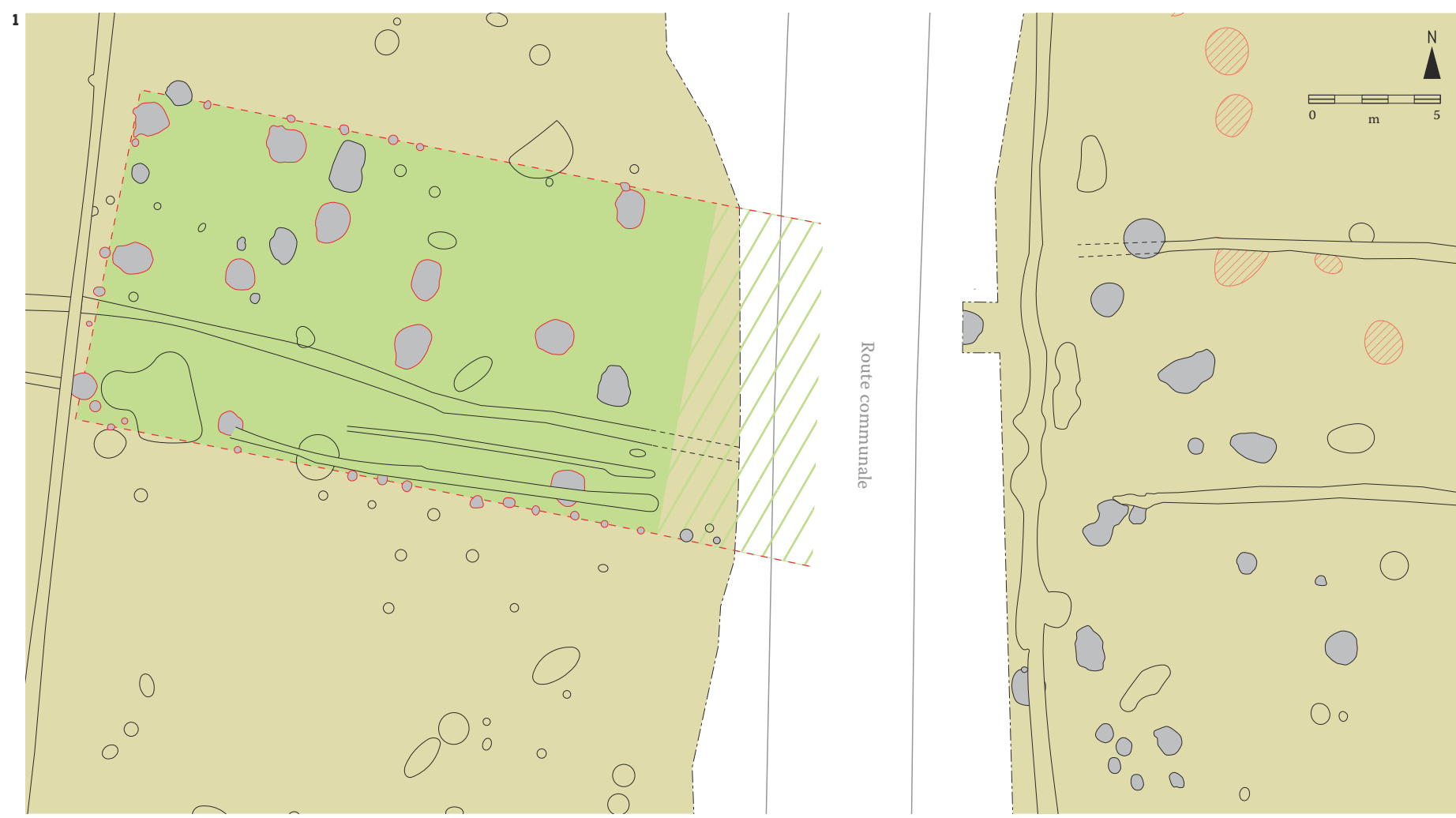

Trous de poteaux liés au bâtiment néolithique Autres structures néolithiques Emprise du bâtiment

Extension probable du bâtiment

1. Plan général de la maison et d'une partie des structures Foyers à pierres chauffées 
constructions plus légères, des fosses, d'autres structures à pierres chauffées ou encore de petits épandages de mobilier céramique ou lithique témoignent d'une forte occupation du secteur au Néolithique moyen. Il faut d'ailleurs souligner qu'un diagnostic réalisé dans les années 1990 (O. Kayser, 1992) en périphérie immédiate du site avait livré des vestiges parfaitement contemporains, ce qui témoigne d'une occupation se développant sur au moins une quinzaine d'hectares.

Au niveau régional, les fouilles récentes comme celles de Guichen et de Pléchatel (fouilles L. Juhel), de Lillemer (fouilles de L. Laporte et C. Bizien) en Ille-et-Vilaine, de Châteauneuf-du-Faou (fouille J.-Y Tinevez) dans le Finistère ont permis de combler de nombreuses lacunes concernant l'habitat au Néolithique moyen. Malgré tout, ce dernier reste très difficile à caractériser et les plans de bâtiments sont encore trop peu nombreux dans une région où, paradoxalement, les monuments mégalithiques sont omniprésents.

La grande maison découverte à La Mézière constitue donc un apport scientifique de premier ordre pour la connaissance del'habitat néolithique dans la péninsule armoricaine. Par ses dimensions importantes, cet édifice pose question quant à sa vocation. S'agit-il d'une structure à usage collectif ou non? Quelle est la nature des activités (domestiques, artisanales...) qui ont pu y être réalisées? On ne trouve pas de véritables points de comparaison dans le Néolithique moyen du nord-ouest de la France. Les quelques constructions sur poteaux actuellement reconnues pour cette période sont de plan rectangulaire, voire circulaire, mais offrent généralement des dimensions plus restreintes, en accord avec des habitations à caractère familial. Il faut alors se diriger vers l'est de la France et de contextes culturels différents pour proposer d'éventuels rapprochements avec des architectures de grande dimension comme celles trouvées à Mairy dans les Ardennes (Marolle, 1989).

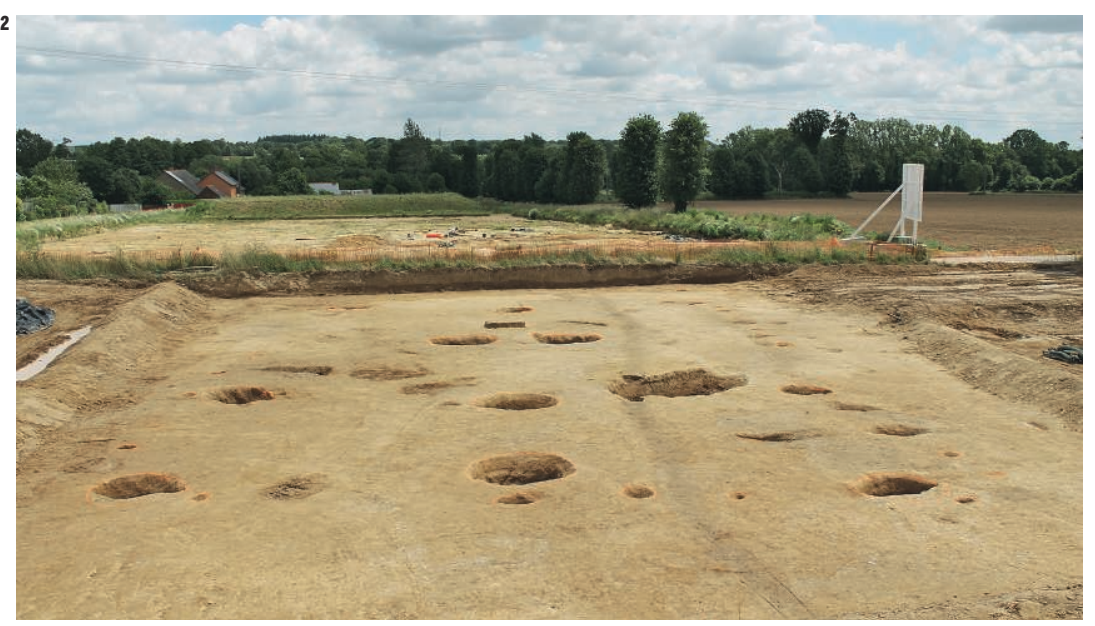

2. Extrémité ouest de la maison. Les structures cerclées en orange correspondent aux trous de poteau qui matérialisent au sol le plan de la construction. II est possible qu'elle se développe jusqu'à la zone fouillée en arrière-plan. 3. Les trous de poteaux porteurs sont de forte dimension. On observe ici parfaitement le négatif du poteau (en gris).

4. Dégagement d'une des structures à pierres chauffées présentes à proximité de

la maison.
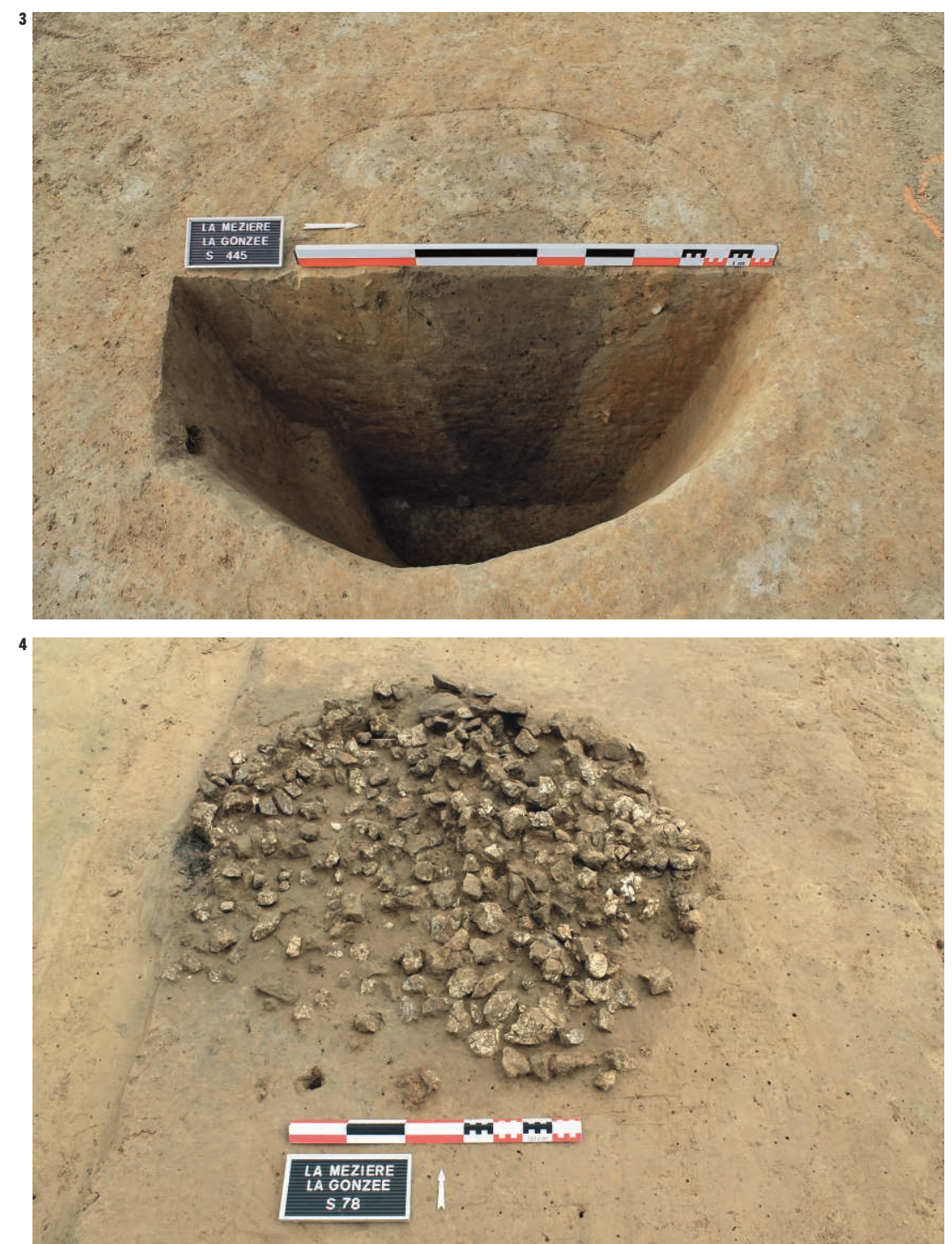

\section{Références bibliographiques}

Escats Y., 2011, La Mézière (Ille-et-

Vilaine), ZAC des Lignes de la Gonzée, Rapport de diagnostic, Inrap, 203 p.

JUHEL L., 2013, Une occupation $d u$ Néolithique moyen et un enclos funéraire du Haut-Empire, Rapport de fouilles, Inrap.

KAYSER O., 1992, La Mézière,Ille-et-Vilaine, site de la Patenotrais, Rapport de sondage, Service régional de l'archéologie.

LAPORTE L. et al., 2003, «Aménagements du Néolithique moyen dans le marais de Dol, au pied de la butte de Lillemer (Ille-et-Vilaine) : les apports d'un programme de prospection thématique ", Revue archéologique de l'Ouest, $\mathrm{n}^{\circ}$ 2o, p. 127-153.

MAROLle C., 1989, « Le village michelsberg des Hautes Chanvières à Mairy
(Ardennes). Étude préliminaire des principales structures », Gallia Préhistoire, t. 31, p. 93-118.

Tinevez J.-Y., 2011, Châteauneuf-duFaou (Finistère), Kermenguy, un habitat du Néolithique moyen, Rapport de fouille programmée Service régional de l'archéologie, $28 \mathrm{p}$. 